\title{
Absolute judgments in speeded tasks: Quantification of the trade-off between speed and accuracy
}

ROBERT G. PACHELLA, Johns Hopkins University, DENNIS F. FISHER and ROBERT KARSH, U. S. Army Human Engineering Laboratories, Aberdeen, Md. 21005

The Ss engaged in making absolute judgments were gradually forced to increase the speed of their responses. Information transmission decreased linearly as criterion time was decreased from $1.0 \mathrm{sec}$ to $0.4 \mathrm{sec}$.

Several papers (Fitts, 1966; Pachella \& Pew, 1968; Smith, 1968) have appeared recently, presenting evidence that a S's latencies in a choice reaction time experiment represent the solution to a fairly complex optimization problem. The average speed of the S's responses appears to be determined by whatever the $S$ (and/or the E) feels is a suitable error rate for the particular experimental situation: the more errors a $S$ is allowed to make, the faster his average reaction time will be. Few of these recent experiments, however, have attempted to quantitatively measure a S's ability to trade speed for accuracy.

Earlier experiments (Hick, 1952; Hyman, 1953) have suggested that average reaction time is linearly related to the amount of information the $S$ is required to transmit. The typical paradigm of these experiments has been to vary the informational content of the stimulus set (e.g., the number of alternative stimuli or the probability of the alternative stimuli) and to record the average reaction time for blocks of trials on which there was errorless performance. Utilizing such a method, reaction time (the dependent variable) is generally expressed as a function of the informational requirements of the task (the independent variable).

It would be of interest, however, to reverse the dependent and independent variables. If one could control the average reaction time of the Ss' responses, would the amount of information transmitted still be linearly related to speed? Such a paradigm would have several advantages over the previous methods. First, the informational requirements or stimulus aspects of the task could remain constant throughout the experiment. Thus, all conditions would be strictly comparable with respect to the stimuli used. Secondly, with stimulus information constant across conditions, the amount of information transmitted by the $S$ becomes a direct measure of the accuracy of his performance: the more errors made by the $S$ and the wider the dispersion of errors (i.e., the less accurate his performance), the less information he transmits. Thus, it would be possible to measure the S's ability to trade speed for accuracy.

The present paper reports an experimental manipulation which satisfactorily controls the speed at which Ss respond. One of the further advantages of this particular method is that it allows tasks which are not normally used in reaction time studies to be gradually shaped into speeded tasks. For example, in the present experiment the $S$ begins with a common absolute judgment task (judging visual position) and is gradually made to work at faster rates of speed until finally the task closely resembles the typical choice reaction time situation. In this way the relationship between choice reaction time experiments and absolute judgment experiments can be more clearly understood.

\section{Subjects} METHOD

Five U. S. Army enlisted men between ages of 18 and 25 , each with at least 20-20 vision (uncorrected) served as Ss.

Procedure

On every trial, in all conditions, Ss were required to make an absolute judgment about which of 10 equally spaced positions a vertical bar marker ( 0.25 in. $x 0.06$ in.) held in a visual field ( 2 in. $x 1.5$ in.). Stimuli were presented at a distance of 16 in. in a Scientific Prototype automatic three channel tachistoscope.

The Ss responded on each trial by pressing one of 10 keys on which their fingers rested. The assignment of keys to visual positions was spatially compatible. Immediately following each response Ss were given visual feedback indicating the correct response. On each trial the stimulus, response and response time were recorded.

The sequence of events on each trial was as follows: A stimulus was presented. The $S$ responded by pressing the key which he judged to correspond to the particular visual position. The response terminated the stimulus and initiated the feedback display which remained visible for $3.1 \mathrm{sec}$. A blank field of $1.5 \mathrm{sec}$ duration followed the feedback and served as a warning signal for the next stimulus presentation. One hundred such trials (each stimulus position occurring 10 times, randomly) constituted one block of data. Following each block the $\mathbf{S}$ was given a short rest period during which he was instructed concerning the speed requirements for the next block of trials.

Speed requirements

After one block of unspeeded absolute judgments, an interval timer of $1.0 \mathrm{sec}$ was introduced into the experiment. This timer was started on each trial with the onset of the stimulus. Ss were instructed to attempt to make all of their responses faster than this $1.0 \mathrm{sec}$ timer and within this time constraint to be as accurate as possible. Whenever a $S$ responded in less than $1.0 \mathrm{sec}$ he received auditory feedback in the form of a $2500 \mathrm{~Hz}$ tone. Slow responses were not acknowledged with auditory feedback. All other aspects of the task were identical to the first block of absolute judgment trials.

Following 100 trials with the interval timer at $1.0 \mathrm{sec}$, the timer was reduced (with the S's knowledge) to $0.7 \mathrm{sec}$. Instructions and feedback about speed were given as in the previous block, except with the criterion time now being $0.7 \mathrm{sec}$.

One last block of 100 trials was run with the interval timer set at $0.4 \mathrm{sec}$. The task, instructions and feedback were all identical to the previous blocks.

\section{RESULTS}

Figure 1 represents the average information transmitted and median reaction time for each of the experimental conditions. The upper point (dashed line) is presented as a limiting value since it represents the amount of information transmitted by Ss in the unspeeded absolute judgment conditions. This value, commonly called channel capacity, represents the maximum discriminating ability of the Ss for the particular stimuli being used. The remaining points represent the varying decrements in discrimination produced by forcing the $S$ to speed his judgments. The speeded judgments produce a nearly perfect linear relationship between information transmitted and reaction time. The function

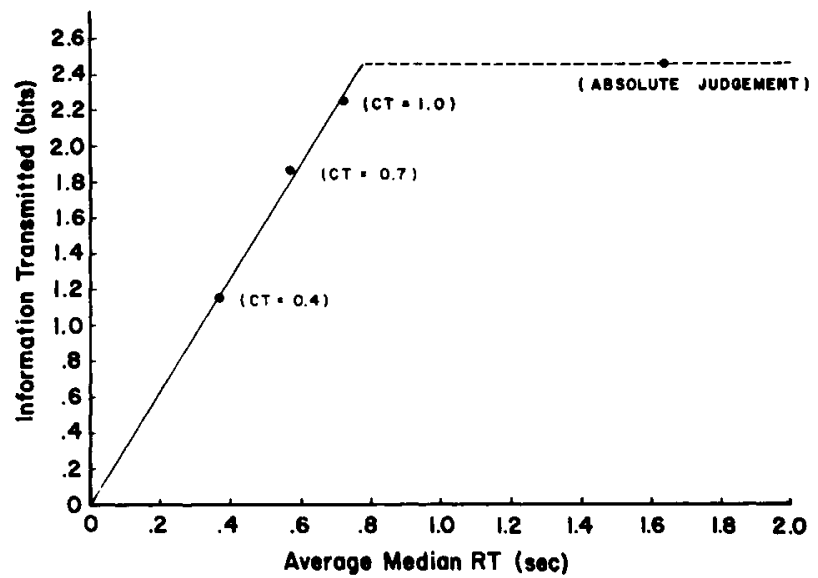

Fig. 1. Information transmission as a function of reaction time for each experimental condition. 


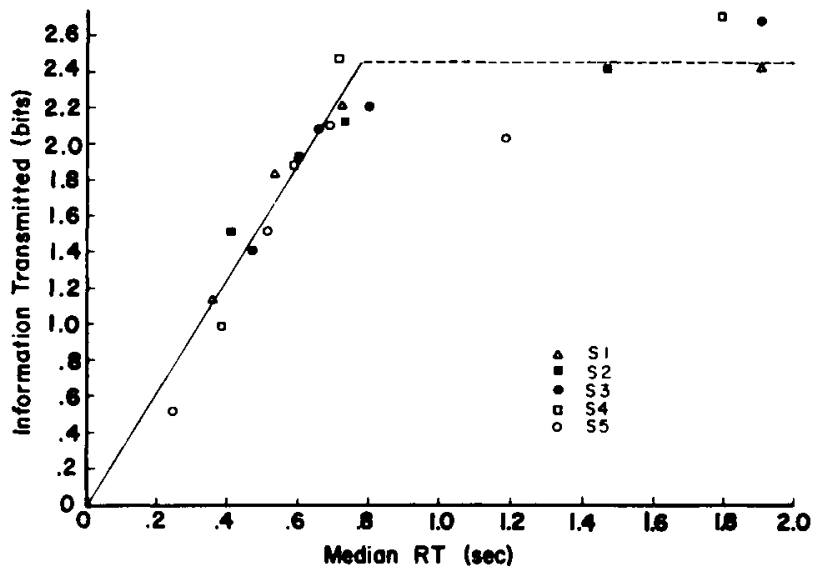

Fig. 2. Information transmission as a function of reaction time for individual Ss.

plotted (slope $=3.1 \mathrm{bits} / \mathrm{sec}$ ) accounts for better than $99 \%$ of the variance in the experimental conditions.

Figure 2 presents evidence that the apparent linearity of the data is not simply a function of the averaging process in grouping the data. Each point in Fig. 2 represents one block of trials for a particular S. All five Ss produced linear data, although one of the five (S 2) produced a large positive intercept indicating that if faster speed constraints had been used his data would have been curvilinear.

\section{CONCLUSION}

Forcing a $S$ to speed his judgments in an absolute judgment experiment causes a decrease in the accuracy of his responses. The relationship between speed and accuracy appears to be that predicted by previous choice reaction time studies: When accuracy is measured by the amount of information transmitted, equal increases in speed will produce equal decreases in accuracy. There is, however, a point beyond which slowing down response speed does not improve the accuracy of performance. This point appears to be determined by the number of absolute identifications the $S$ is capable of making. This last fact is generally overlooked in reaction time experiments since the stimuli usually employed in such studies are perfectly discriminable.

\section{REFERENCES}

FITTS, P. M. The cognitive aspects of information processing III: Set for speed vs accuracy. Journal of Experimental Psychology, 1966, 71, 949-857.

HICK, W. E. On the rate of gain of information. Quarterly Journal of Experimental Psychology, 1952, 4, 11-26.

HYMAN, R. Stimulus information as a determinant of reaction time. Journal of Experimental Psychology, 1953, 45, 188-196.

PACHELLA, R. G., \& PEW, R. W. Speed-accuracy trade-off in reaction time: The effect of discrete criterion times. Journal of Experimental Psychology, $1968,76,19-24$.

SMITH, E. E. Choice reaction time: An analysis of the major theoretical positions. Psychological Bulletin, 1968, 69, 77-1 10. 